\title{
Pulsing Policies for Aggregate Advertising Models
}

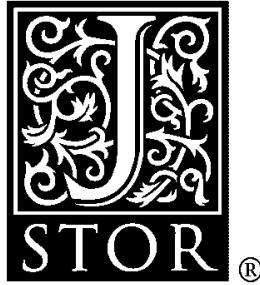

Fred M. Feinberg

Marketing Science, Vol. 11, No. 3. (Summer, 1992), pp. 221-234.

Stable URL:

http://links.jstor.org/sici?sici=0732-2399\%28199222\%2911\%3A3\%3C221\%3APPFAAM\%3E2.0.CO\%3B2-\%23

Marketing Science is currently published by INFORMS.

Your use of the JSTOR archive indicates your acceptance of JSTOR's Terms and Conditions of Use, available at

http://www.jstor.org/about/terms.html. JSTOR's Terms and Conditions of Use provides, in part, that unless you have obtained prior permission, you may not download an entire issue of a journal or multiple copies of articles, and you may use content in the JSTOR archive only for your personal, non-commercial use.

Please contact the publisher regarding any further use of this work. Publisher contact information may be obtained at http://www.jstor.org/journals/informs.html.

Each copy of any part of a JSTOR transmission must contain the same copyright notice that appears on the screen or printed page of such transmission.

JSTOR is an independent not-for-profit organization dedicated to and preserving a digital archive of scholarly journals. For more information regarding JSTOR, please contact support@jstor.org. 


\title{
PULSING POLICIES FOR AGGREGATE ADVERTISING MODELS
}

\author{
FRED M. FEINBERG \\ Duke University
}

Classical continuous-time models of advertising expenditure tend to fall into two categories, those that prescribe spending at a constant level and those that prescribe switching infinitely quickly between several different levels of spending. The latter practice, chattering, though impossible literally, can be interpreted in practice to imply that the faster the switching the better. Empirical evidence, however, sometimes suggests the superiority of pulsing, alternating between different spending levels with finite frequency, for example in a periodic fashion. Furthermore, the actual behavior of marketing managers, who often advertise in flights or pulses, appears to differ from the optimal policies many current models prescribe.

Showing how structural properties of common advertising models rule out finite-frequency pulsing a priori, we develop a continuous-time model for which finite-frequency pulsing can be optimal. Relaxing these structural assumptions yields a new class of models which, for certain values of their parameters, lead to periodic pulsing optima; this is accomplished by inclusion of both S-shaped response and an exponential-smoothing filter. These theoretical results are illustrated through simulation of a variant of the Vidale-Wolfe model.

(Advertising; Optimal Control; Pulsing)

\section{Introduction}

While numerous articles address the formulation and solution of advertising expenditure problems, after 30 years of research, questions of an elemental nature stand only partially answered. One such question concerns the best way to allocate a given advertising budget over time, whether it is best to spend it as evenly as possible, to alternate periods of greater spending with periods of relatively lesser spending, or to do something more subtle. Methods to choose between these options have offered mixed conclusions, often relying strongly on logical assumptions about how consumers respond as a group to changes in advertising spending. Empirical evidence has often favored spreading advertising funds evenly or implied the superiority of such a scheme, though the results can't be called conclusive (Zielske 1959, Rao 1970, Ackoff and Emshoff 1975, Rao and Miller 1975, Simon and Arndt 1980, Eastlack and Rao 1986, Steiner 1987).

We will focus on a set of deterministic, continuous-time models whose members can be broadly characterized. For such models, the optimal long-run method for allocating advertising funds over time has tended to be to hold sales constant by one of two methods: holding advertising at a constant level, or switching infinitely quickly between two different levels. The practice of infinitely rapid switching, or chattering, offers no believable advice to the practitioner, implying as it does an increase in profit for every increase in pulsing 
frequency. In short, neither chattering nor constant spending is wholly satisfying; each precludes a possibility that may work better, pulsing, advertising for a while at one level, then moving on to another, and yet another, so long as the switching occurs with finite frequency. To the extent that pulsing might be a realistic and relatively profitable method of allocating advertising funds, any model which renders it automatically inferior may well be missing an essential empirical feature.

We examine a generalization of several well-known models, showing how structural assumptions effectively disallow pulsing optima. Two useful constructs, S-shaped response and Filters, are introduced and, based on these, a class of continuous-time models is presented for which optimal periodic long-run policy can have finite frequency. We first present an overview of general issues relevant to advertising modeling; more detailed treatments can be found in Sethi (1977), Little (1979) or Mahajan and Muller (1986).

\section{Problem Formulation}

To formally define a modeling framework, we assume that the firm's advertising spending rate, $a(t)$ ( measured in dollars/time), determines its sales rate, $s(t)$ (measured in units/time). There is a fixed per-unit production $\operatorname{cost} c$, and each unit sells for a fixed price, $p$. The firm can therefore expect an instantaneous profit rate given by $(p-c) s(t)$ - $a(t)$. Additionally, there is a maximum potential sales rate, $m$; by definition, $s(t)$ $\leq m$. Without loss of generality, $(p-c)$ can be scaled to unity; defining $x(t)=s(t) / m$ and $u(t)=a(t) / m$, we can refer without ambiguity to the scaled profit rate, $\pi(t)$ :

$$
\pi(t)=\frac{(p-c) s(t)}{m}-\frac{a(t)}{m}=x(t)-u(t) .
$$

Note that $x(t)$ is constrained between 0 and 1 and $u(t)$ remains nonnegative, though unbounded. Since we have merely performed a rescaling by constant factors, no clarity is lost by referring to $u$ as advertising, $x$ as sales and $\pi$ as profit; the intermediate variables $(s, a, p, c)$ are no longer necessary.

The relationship between sales $x$ and advertising $u$ is ordinarily captured by a firstorder ODE:

$$
\begin{gathered}
\dot{x}=g(x, u), \\
x(0)=x_{0} .
\end{gathered}
$$

It is possible to modify $g$ to include temporal trends as $g(x, u, t)$, or to include additional arguments for added flexibility. Uncertainty, unobservables and inaccuracy of measurement can be also accounted for by stochastic processes (Tapiero 1978, 1979, 1982; Monahan 1983; Nguyen 1985). Here we restrict our attention to $g(x, u)$ as above.

\section{Steady-State and Periodic Equilibria}

We address the advertising expenditure problem as put forth by Sasieni (1971), the maximization of a discounted profit integral, subject to a state equation and initial condition, of the following type:

$$
\begin{gathered}
\int_{0}^{\infty}(x-u) e^{-r t} d t, \\
\dot{x}=g(x, u), \\
x(0)=x_{0} .
\end{gathered}
$$

We shall call ( 3 ) a Sasieni model. The optimal solution of systems like (3) ordinarily consists of two components (Gould 1970, Sasieni 1971, Sethi 1977): 
A Start-Up Period. A period of finite duration, during which advertising is manipulated so as to bring sales to a specific level at the end of the period.

Long-Run Optimum. The value to which sales have been driven is maintained thereafter by one of two methods: holding advertising constant, or having advertising switch infinitely quickly between two levels. ${ }^{1}$

Though the dynamics of the initial start-up period is of interest in itself, as it is of finite duration, its importance in the long run is heavily dependent on the discount rate, $r$. When $r$ is high, funds made early on are crucial; when $r$ is low, such funds represent but a small fraction of the anticipated discounted profit stream. We focus our attention here on long-run dynamics. ${ }^{2}$

Holding $x$ constant implies $\dot{x}$ vanishes asymptotically, so the defining equation for the long-run sales response function, $x_{\infty}(u)$, is as follows for any constant level of advertising, $u$ :

$$
g\left(x_{\infty}(u), u\right)=0 .
$$

The function $x_{\infty}(u)$ sets up a correspondence between levels of advertising and levels of sales, irrespective of the dynamics of the problem, in effect telling what sales would be if one advertised at $u$ forever. Disregarding discounting, the relation (4) allows univariate maximization over $u$ for the best constant long-run policy.

The steady-state equilibrium defined implicitly by (4) can serve as a benchmark against which the relative profitability of other advertising policies can be measured. An advertising policy is periodic if $u(t+\tau)=u(t)$ for some positive $\tau$; if $\tau$ is the smallest number for which the definition holds, then $u$ is said to be periodic with period $\tau$. Constant spending policies almost fit the definition for periodicity, failing in that there is no uniquely smallest $\tau$ to qualify as a period. Pulsing policies describe finite-frequency periodic spending for which $0<\tau<\infty$; such policies pulse between different levels of advertising spending in a "noticeable" way, neither infinitely slowly nor infinitely fast. A chattering policy then arises as $\tau$ becomes small, and frequency increases without bound.

Formal mathematics aside, we address the following problem: Disregarding a possible finite start-up period, which policy produces the maximum long-run profits, constant spending, pulsing or chattering, and what properties of a model determine its having one or another of these as its optimal solution? ${ }^{3}$

Viewpoints on the expenditure problem split early on into two distinct substreams, corresponding to the differing mechanisms introduced by Vidale and Wolfe (1957) and by Nerlove and Arrow (1962). The Vidale-Wolfe model proposes that advertising directly persuades potential customers not currently buying from the firm, while those who are buying tend to forget (buy less) over time. This is cast in terms of a first order ODE linear in both $x$ and $u$ :

$$
\dot{x}=g(x, u)=\rho u(1-x)-\delta x .
$$

When $u$ is held constant, the solution to $(5)$ is given by:

$$
x(t)=x_{0} e^{-(\rho+\delta u) t}+[\delta u /(\rho+\delta u)]\left[1-e^{-(\rho+\delta u) t}\right] .
$$

\footnotetext{
' It is also possible that the optimal sales path approaches the long-run optimum asymptotically, never explicitly reaching it.

${ }^{2}$ We will subsequently argue that, at periodic equilibrium, discounting can be adequately accommodated outside the profit integral, and can be legitimately sidestepped.

${ }^{3}$ These three options in no way exhaust all possibilities for spending policies; there is plainly a large class of functions which are neither periodic nor constant. Although these technically require consideration, they play a peripheral role in comparing the performance of the kinds of policies which tend to be optimal.
} 
Thus, the initial state condition, $x(0)=x_{0}$, is exponentially phased out, while a longrun level, $x_{\infty}(u)=\delta u /(\rho+\delta u)$, is exponentially phased in. Such exponential motion is an important feature of the Vidale-Wolfe model as well as several others (Ozga 1960, Mesak 1985, Mahajan and Muller 1986).

Nerlove and Arrow (1962) formalized the notion of consumers "building up" a stock of good feeling towards a firm through protracted exposure to the firm's advertising. This stock, termed Goodwill, is then fed into a demand function to produce sales. Goodwill $(G)$ evolves deterministically according to the state equation:

$$
\dot{G}=u(t)-k G \text {. }
$$

Much as in the Vidale-Wolfe model, $G$ moves exponentially as the control $u$ changes from one constant value to another. This exponential dynamic suggests a commonality of operation between $u(t)$-based and Goodwill mechanisms, different though they might outwardly appear.

Optimal policies for both these models and numerous variants have been worked out, ordinarily through control theory or dynamic programming (Gould 1970; Sethi 1973, 1977, 1979). Sasieni (1971), however, closed the book on the optimal policies of a large class of models with seemingly sensible properties. Casting the dynamic problem as in (3), he shows that if the function $g$ satisfies the conditions:

$$
g_{u}>0, \quad g_{x}<0, \quad g_{u u} \leq 0,
$$

then the optimal long-run policy is constant-spending; this reasoning applies to both the Vidale-Wolfe and Nerlove-Arrow specifications. The third condition, $g$ being concave in $u$, reflecting decreasing marginal returns to advertising spending, can hardly be called well entrenched. While a review paper (Simon and Arndt 1980) finds scant evidence for discarding it, a recent model is based largely on its rejection (Mahajan and Muller 1986). Concavity of the response function $g$ rules out the optimality of finite-frequency pulsing. While there is no real argument that $g$ should be asymptotically concave (or at least not asymptotically convex), it is often conjectured, and supported by certain empirical findings, that $g$ should be S-shaped in $u$, so that marginal returns to advertising increase for some period, after which they forever decrease; Simon and Arndt (1980) supply a survey of relevant empirical work.

Referring to Figure $1, g$ is convex in $u$ until an inflection point is reached, after which it becomes concave. Advertising on the convex portion of the curve is suboptimal, since such a policy can be dominated by chattering between $u=0$ and $u=u_{h}$, the tangency point, to produce a superior level of response. If the optimal long-run spending level falls between 0 and $u_{h}$, then one should chatter between those two values; if the optimal level falls beyond $u_{h}$, then one should advertise there in a constant fashion. This method allows questions about chattering to be answered by eliminating the convex portion of $g$, then re-solving the problem by maximizing over $u$ in a univariate fashion.

Other methods allow a different vantage point on the pulsing problem from that pursued here. Deal (1979) analyzes a more general dynamic game, a duopolistic analog of the

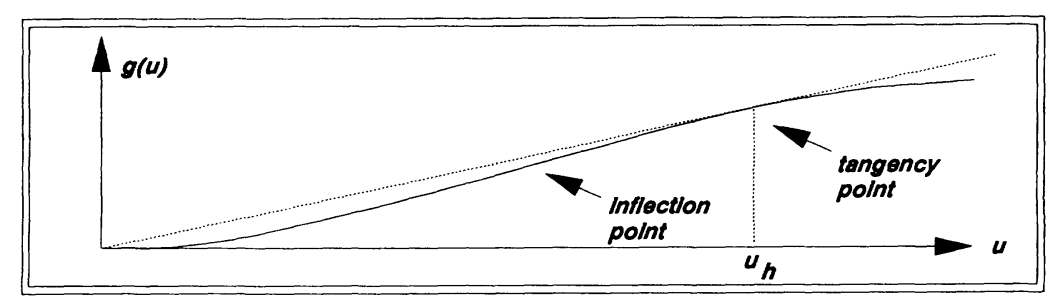

FIGURE 1. S-Shaped Response. 
Vidale-Wolfe model; this work has been generalized to duopolistic Sasieni models by Jones (1983). General oligopolistic dynamics, based on a Goodwill-type mechanism, is addressed by Friedman (1983) and Rao (1984). Both Simon (1982), who argues that classical models are misspecified a priori, and Mesak (1985) have proposed discretetime models in which response asymmetry allows pulsing optimality. Based on an extension of Sasieni's work by Hartl (1987), Luhmer et al. (1988), using a higher-order differential system, have extended Simon's model to continuous time. Although several of these models can allow pulsing optima, each requires a departure from Sasieni's framework.

\section{Discounting and Periodic Equilibrium}

Analysis of the system (3) can be simplified when it is considered at periodic equilibrium, as shown for a rectangular pulsing policy in Figure 2. As equilibrium is approached, the path of $x$, and consequently that of $\pi=x-u$, settles into a functional limit of period $\tau$, so that the undiscounted profit made from period to period approaches a constant value. If discounting is then desired, it can be accomplished discretely, each period's profit contribution equal to the previous period's corrected by a factor of $e^{-r \tau}$. The result is a geometric series, with total profit on $[0, \infty]$ equal to $\left(1-e^{-r \tau}\right)^{-1}$ times profit made in a single period. Thus, for any periodic $u(t)$, maximizing total discounted profit over the infinite horizon is equivalent to profit maximization over a single period at equilibrium (a strategy used by Thompson 1981). Since the profits made in a potential "start-up" period are seen as less important the smaller the value of $r$, focusing on long-run dynamics, as we do, is similar to a focus on small $r$. Because a small discount rate is not radically different from no discount rate, long-run optimal strategies of systems like (3) can be characterized by studying a simpler problem, that of maximizing profits over a single period at periodic equilibrium.

\section{Local Analysis of General Models}

It is possible to apply periodic policies to general dynamical systems like (3), linking the possibility of pulsing optima to local conditions of $g(x, u)$. Sasieni's proof of the superiority of constant spending or chattering depends on chattering out an S-shaped $g$ to its convex hull, by chattering in the form of a rectangular wave between $u=0$ and $u$ $=u_{h}$. We present a simple proof that, at periodic equilibrium and with concave $g$, if chattering is to be optimal, the "shape" of such chattering (considered as a functional limit as $\tau \rightarrow 0$ ) must be constant spending. This will require, as does Sasieni's proof, the partial condition $g_{u u}<0$. Suppose the system (3) is held at periodic equilibrium by pulsing as $u(t)$, so that $x(\tau)=x(0)$; as the pulse period $\tau$ approaches 0 , this induces a constant sales level $x^{*}$ :

$$
0=\frac{x(\tau)-x(0)}{\tau}=\lim _{\tau \rightarrow 0} \frac{1}{\tau} \int_{0}^{\tau} g\left(x^{*}, u(t)\right) d t .
$$

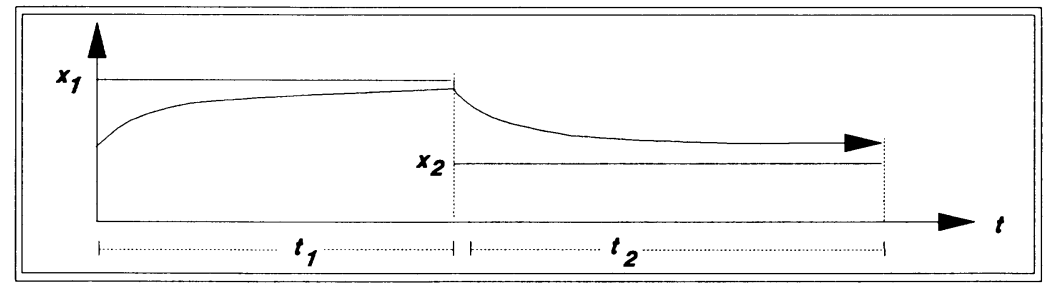

Figure 2. Response to Rectangular Pulse at Periodic Equilibrium. 
For clarity, we treat the integral alone, ignoring the external limit. We ask what $u(t)$ maximizes $x^{*}$. This can be facilitated by the calculus of variations, defining a new function as the deviation of $u(t)$ from its average value:

$$
\xi(t)=u(t)-\bar{u}
$$

If $u(t)$ is optimal, then $x^{*}$ in the following is maximized when $w=1$ :

$$
\int_{0}^{\tau} g\left(x^{*}, \bar{u}+w \xi(t)\right) d t=0 .
$$

Chattering can only be optimal if $\xi(t)$ is not identically zero on $[0, \tau]$ and if the pair ( $w$, $\xi(t))$ are optimal when $w=1$. One can therefore differentiate (11) inside the integral with respect to $w$ :

$$
\int_{0}^{\tau} g_{x}\left(x^{*}, \bar{u}+w \xi(t)\right)\left(\partial x^{*} / \partial w\right) d t+\int_{0}^{\tau} g_{u}\left(x^{*}, \bar{u}+w \xi(t)\right) \xi(t) d t=0 .
$$

At $w=1, \partial x^{*} / \partial w$ must be 0 for $x^{*}$ to be maximized; so, recalling the definition for $\xi(t)$, (12) becomes:

$$
\begin{gathered}
\int_{0}^{\tau} g_{u}\left(x^{*}, u(t)\right) \bar{u} d t=\int_{0}^{\tau} g_{u}\left(x^{*}, u(t)\right) u(t) d t, \\
E\left[g_{u}\left(x^{*}, u(t)\right)\right] E[u(t)]=E\left[g_{u}\left(x^{*}, u(t)\right) u(t)\right], \\
\operatorname{Cov}\left[g_{u}\left(x^{*}, u(t)\right), u(t)\right]=0 .
\end{gathered}
$$

When $g_{u}$ is decreasing in $u$ (i.e., $g_{u u}<0$ ), the covariance condition (14) can be satisfied only if $u(t)$ is constant. Thus, constant spending is superior to any type of chattering when $g$ is concave in $u$.

\section{A Model with Finite-Frequency Periodic Pulsing Optimum}

Given a Sasieni model (3), the superiority of either chattering or of constant spending is automatic. Local properties of $g$ favor constant spending when $g$ is concave and chattering when $g$ is S-shaped, while linear response equates chattering with constant spending, making them perform equally well and better than all intermediate pulsing policies. Nerlove-Arrow Goodwill has the property that all periodic inputs of equal average value have the same average Goodwill output, in the long run. We show that a common smoothing technique is similar to Goodwill in this regard, in effect filtering out incoming chattering inputs. When coupled with a suitably S-shaped $g$, a model with a finite-frequency periodic optimum can result.

\section{Filters and Their Properties}

A feature of the Sasieni model formulation (3) is that profit can differ for constant spending and chattering policies of the same average value. There is merit to the argument that the only way to produce something appearing constant within the confines of an inherently discrete medium like advertising is to fluctuate very rapidly, that is, to chatter. An analogy here is that of alternating current; while a light blinking on and off every second or so is irritatingly noticeable, one cycling at 60 flashes per second is practically indistinguishable from one on all the time. Since chattering is impossible in principle and constant spending is impossible in practice (unless one pays for an endless commercial), one might say that the two are unrealizable ends of a frequency spectrum and are, in a sense, perceptually equivalent. Filters allow us to mathematically equate the two; passing a chatter through a filter changes it to a constant policy. However, filtering 
a "noticeable" periodic pulsing policy (one of moderate frequency) yields as output, at equilibrium, a different periodic policy, though with unchanged period and average level.

A useful method for exponentially smoothing out rapidly fluctuating functions (or continuizing discrete ones) operates through the differential equation:

$$
\dot{z}=\eta(u-z) \text {. }
$$

Specifications equivalent to (15) have been used widely in economics, notably in partial adjustment and distributed lag models. Koyck (1954) identifies subjective reasons for lags, such as psychological inertia, and objective reasons, such as those related to technological constraints. Nerlove (1958), in work pre-dating the Nerlove-Arrow model, studies supply dynamics through an analog to (15). Distributed lag models have frequently been applied to advertising-sales data, as in Bass and Clarke (1972) and in Clarke (1976), while Vanhonacker (1983) discusses these issues and applies a partial adjustment model to amend model misspecifications. Rao (1986) surveys much relevant work in marketing, focusing on estimation in continuous-time, specifically utilizing a differential process based on the Nerlove-Arrow model. A comprehensive treatment of distributed lag models and their estimation appears in Dhrymes (1981).

We conceptualize advertising as affecting sales through the unobservable $z$, deterministically related to the actual advertising path $u$. A partial adjustment mechanism, perhaps driven by consumer inertia or household level consumption, can give rise to such a filtering process in aggregate. In light of Hartl (1987), who rules out first-order models as an explanation for pulsing, a specification like (15) appears sensible, linking advertising to a second-order differential process.

Solving (15) via an integrating factor is straightforward. In the appendix, we demonstrate the following facts about filters operating at periodic equilibrium:

(1) The average level of the pulsing policy, $u$, is unchanged over $[0, \tau]$ :

$$
\int_{0}^{\tau} z(t) d t=\int_{0}^{\tau} u(t) d t
$$

(2) As $\eta$ approaches infinity, $z$ approaches $u$ pointwise, so long as $u(t)$ is bounded on $[0, \tau]$ (i.e., $\eta$ can be construed as a rate of adjustment between the equilibrium paths of $u(t)$ and $z(t))$.

(3) If $u(t)$ is a chattering policy, the resulting $z(t)$ will be constant on $[0, \tau]$.

\section{A Class of Models with Pulsing Optima}

Here we show that filtering, in conjunction with S-shaped response, can produce finitefrequency pulsing optima; the proof is structured as in Figure 3. Given the space of periodic inputs to the Sasieni model (3), one can decompose the $u(t)$ 's into a oneparameter family, all $u$ 's within a single class sharing the same average value. Within a particular class, any $u$ induces a $z$ of the same average level when put through the filter (15), but this $z$ could not be chattering. If the resultant $z$ 's were then fed into a model favoring pulsing over constant spending, a finite-frequency $u$ would have to dominate

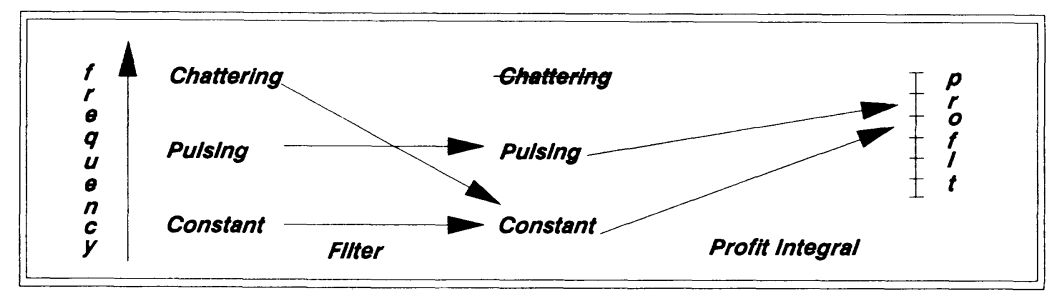

FIGURE 3. Outline for Proof of Finite-Frequency Optimum. 
both a chattering $u$ and a constant $u$, since the filter turns these last two into constant spending, which by hypothesis is not optimal. Within any of the original classes of $u$ 's (here indexed by their average level), since neither chattering nor constant spending can be optimal, they cannot be optimal overall, demonstrating the global superiority of a single finite-frequency pulsing policy.

Finding a model for which pulsing is superior to constant spending requires making $g(x, u)$ suitably $\mathrm{S}$-shaped. This suggests a system of the following type (disregarding discounting), where the goal is to maximize $\pi_{N}[u(t)]$, nonfiltered profit ( $N$ refers to nonfiltered):

$$
\begin{gathered}
\pi_{N}[u(t)] \doteq \frac{1}{\tau} \int_{0}^{\tau}[x(t)-u(t)] d t, \\
\dot{x}=g(x, u) .
\end{gathered}
$$

Assume $g$ is S-shaped in such a way that the optimal solution is to chatter between $u=0$ and $u=u_{h}$, the point where $g$ first meets its convex hull. With $g$ unchanged, consider the system with an added filter, defining filtered profit $\pi_{F}[u(t)](F$ refers to filtered $)$ :

$$
\begin{aligned}
\pi_{F}[u(t)] \doteq \frac{1}{\tau} \int_{0}^{\tau}[x(t)-u(t)] d t, \\
\dot{x}=g(x, z), \\
\dot{z}=\eta(u-z) .
\end{aligned}
$$

It is crucial to note that, although chattering is assumed optimal for the nonfiltered system, it cannot be the unique optimum for the filtered system, since a constant policy equal to the average level of the chatter does just as well. We will assume that, as in Sasieni's formulation, there is some pulsing policy which, relative to the nonfiltered system, performs better than constant spending, but not so well as chattering. Using the subscripts $c$ for a chattering policy, $p$ for a pulsing policy and $k$ for a constant policy, we have the following correspondence as $\eta$ tends to infinity: ${ }^{4}$

$$
\begin{aligned}
& u_{k} \rightarrow z_{k}, \\
& u_{p} \rightarrow z_{p}, \\
& u_{c} \rightarrow z_{k} .
\end{aligned}
$$

THEOREM. If $u_{c}$ maximizes $\pi_{N}$, then some $u_{p}$ maximizes $\pi_{F}$.

ProOF. By assumption, $g$ is such that there exists a finite-frequency pulsing policy, $u_{p}$, which, when compared with chattering or a constant policy of the same average level, performs worse than the chatter and better than the constant for the nonfiltered system:

$$
\pi_{N}\left(u_{c}\right)>\pi_{N}\left(u_{p}\right)>\pi_{N}\left(u_{k}\right) .
$$

Then (19) implies:

$$
\begin{aligned}
& \pi_{F}\left(u_{k}\right)=\pi_{N}\left(z_{k}\right), \\
& \pi_{F}\left(u_{p}\right)=\pi_{N}\left(z_{p}\right), \\
& \pi_{F}\left(u_{c}\right)=\pi_{N}\left(z_{k}\right) .
\end{aligned}
$$

\footnotetext{
${ }^{4}$ An additional requirement on the dynamic system (17) is necessary: If a family of inputs, $\left\{z_{\alpha}\right\}$ (where $\alpha$ runs over some index set), converges pointwise to a functional limit, $z$, then the objective values $\pi_{N}\left[z_{\alpha}\right]$ converge to $\pi_{N}[z]$, a reasonable requirement on $g$ considering that these are models of advertising.
} 
We can compare the performance of pulsing to that of chattering and constant spending for the filtered system:

$$
\pi_{F}\left(u_{p}\right)=\pi_{N}\left(u_{p}\right)>\pi_{N}\left(u_{k}\right)=\pi_{F}\left(u_{k}\right)=\pi_{F}\left(u_{c}\right) .
$$

Since this inequality holds as $\eta$ tends toward infinity, it holds for large finite values of $\eta$ as well. Thus, given a Sasieni-model with optimal chattering, the optimal policy for the related filtered model, provided $\eta$ is large enough, is finite-frequency pulsing. The sense of this result is that, when advertising effects are taken to both follow a type of partial adjustment (as they might when current sales alone do not fully capture the effects of past advertising on sales rate changes) and display short-run convexity (a situation for which empirical evidence exists in some product classes), it may be that it is optimal, in the long-run, for advertising spending to be pulsed.

We note that optimal policy is not necessarily of a type that changes abruptly between piecewise-flat levels, and the shape of the policy depends on the specific qualities of $g$. Moreover, as there is no guarantee that $u$ stays bounded between 0 and $u_{h}$, finding the optimum by numerical techniques can prove difficult.

\section{Numerical Example and Simulation}

We present a simulation of a modified Vidale-Wolfe model, with and without the additional filter variable, $z$. Following Mahajan and Muller (1986), we specify

$$
\dot{x}=\rho f(u)(1-x)-\delta x,
$$

where $f(u)$ is chosen from the flexible family of curves

$$
f(u)=u^{\alpha}\left(\gamma+u^{\beta}\right)^{-1} .
$$

Depending on its parameters, $f(u)$ can be linear, strictly convex or concave, or S-shaped; we are interested in the last of these possibilities. Differentiation verifies that $f$ is S-shaped and twice-differentiable when $\alpha \geq 2, \alpha-1 \leq \beta \leq \alpha$ and $\gamma>0$. If we also set $f^{\prime}\left(u_{h}\right)=1$ for convenience in subsequent maximization, the following is a suitable choice of parameters:

$$
\alpha=\frac{5}{2}, \quad \beta=2, \quad \gamma=3^{3} / 4^{4} .
$$

The S-shaped $f$ defined by these parameters implies that the point where $f$ meets its convex hull is $u_{h}=\frac{9}{16}$; further, $f\left(u_{h}\right)=\frac{9}{16}$ and $f^{\prime}\left(u_{h}\right)=1$. Setting $\rho=4$ and $\delta=1$, it follows from univariate maximization that the optimal chattering policy involves chattering between $u=0$ and $u_{h}=\frac{9}{16}$, spending $\frac{4}{9}$ of the time at the upper value. At optimum we find that $\bar{u}=0.25, x=0.5$ and, consequently, profit $\pi=0.25 .^{5}$

We should compare this to the optimal constant spending policy, that is:

$$
\max _{u} x_{\infty}(u)-u
$$

for the model (23)-(25). This, like the chattering case, requires a univariate maximization, though numerical methods must be used to solve the resulting equations. The optimal constant spending policy sets $u=0.3507, x=0.5605$ and $\pi=0.2098$. As expected, since $f(u)$ is S-shaped, the optimal chattering policy is superior to the optimal constant spending policy or, as Sasieni shows, to any finite-frequency pulsing policy.

We consider this example in the sense of the previous section, that is, relative to a filtered system. Since a numerical search will be necessary, we attempt to show that the

\footnotetext{
${ }^{5}$ Calculating the optimal chattering policy amounts to replacing the concave portion of $f(u)$ with a line from $(0,0)$ to the tangency point, as pictured in Figure 1 . The mechanics for doing so is straightforward and carried out by Mahajan and Muller (1986).
} 
TABLE 1

Optimal Rectangular Policies for Different Values of Filter Parameter $\eta$

\begin{tabular}{rccccc}
\hline$\eta$ & $\tau$ & $\bar{u}$ & $p$ & $\pi_{N}$ & $\pi_{F}$ \\
\hline 6 & 1.132 & 0.293 & 0.518 & 0.2331 & 0.2104 \\
8 & 1.067 & 0.287 & 0.510 & 0.2349 & 0.2134 \\
12 & 0.988 & 0.279 & 0.496 & 0.2377 & 0.2191 \\
16 & 0.911 & 0.272 & 0.485 & 0.2397 & 0.2238 \\
20 & 0.849 & 0.268 & 0.478 & 0.2410 & 0.2272 \\
25 & 0.776 & 0.264 & 0.472 & 0.2422 & 0.2301 \\
30 & 0.731 & 0.262 & 0.468 & 0.2433 & 0.2323 \\
40 & 0.672 & 0.260 & 0.464 & 0.2443 & 0.2352 \\
50 & 0.617 & 0.259 & 0.461 & 0.2454 & 0.2372 \\
60 & 0.573 & 0.258 & 0.458 & 0.2459 & 0.2386 \\
75 & 0.527 & 0.257 & 0.455 & 0.2463 & 0.2401 \\
100 & 0.485 & 0.256 & 0.452 & 0.2468 & 0.2423 \\
\hline
\end{tabular}

optimal rectangular pulsing policy for the following system is neither chattering nor constant spending:

$$
\begin{gathered}
\max _{u(t)} \frac{1}{\tau} \int_{0}^{\tau}[x(t)-u(t)] d t, \\
\dot{x}=\rho f(z)(1-x)-\delta x, \\
\dot{z}=\eta(u-z),
\end{gathered}
$$

where $\rho=4, \delta=1, f(u)=u^{\alpha} /\left(\gamma+u^{\beta}\right), \alpha=2.5, \beta=2, \gamma=3^{3} / 4^{4}$. This specifies all parameters but the filter parameter, $\eta$, which we let assume a variety of values.

The differential system (27), both with and without the filter, was simulated in the MATLAB system using second- and third-order Runge-Kutta methods, with error tolerance set to one part in $10^{6}$. Rectangular pulsing policies (e.g., Figure 2) were fed as inputs $u(t)$; where theory can be used to calculate $x$ and $\pi$ from $u$ (i.e., when there is no filter), simulation results accord remarkably well with theoretical values.

A rectangular pulsing policy is defined by four quantities: $\tau$, its period length; $\bar{u}$, the average level of the pulse; $p$, the proportion of time spent at the higher value; and $u_{\mathrm{dev}}$, the downward deviation of the lower part of the pulse from $\bar{u}$. Preliminary runs indicated that, at optimum, $u_{\mathrm{dev}}$ should be set as close as possible to $\bar{u}$, so all results have been fixed at $u_{\mathrm{dev}}=0.999 * \bar{u} .{ }^{6}$ To locate the optimal rectangular policy for each value of $\eta$, a grid search was run for $\{\tau, \bar{u}, p\}$, and simulated long enough for the system (27) to approach equilibrium; the system was considered close to equilibrium when profit changed by no more than one part in $10^{4}$ with each successive pulse. Table 1 lists the optimal rectangular policies for 12 different values of $\eta,\{6,8,12,16,20,25,30,40,50,60,75$, $100\}$; this information is displayed graphically in Figure $4 \mathrm{a}-\mathrm{d}$. Simulations setting $\eta$ to integral values less than 6 indicated that constant spending was apparently optimal for such values of $\eta$.

The results of Table 1 accord well with theory. As the value of the filter parameter $\eta$ increases, the resulting $z$ will be ever closer to the input $u$, so input policies approaching chattering will fare better. Thus, we see period length $\tau$ becoming smaller, the average

\footnotetext{
${ }^{6}$ Compared with the more straightforward method of listing the levels and durations of each of the pulse's two legs, the present method allows easy comparison of pulses of the same average level. Although setting $u_{\mathrm{dev}}$ $=\bar{u}$ may appear more sensible, setting $u_{\mathrm{dev}}=0.999 * \bar{u}$ guards against the simulation "overshooting" and, erroneously, allowing negative values of $z$.
} 


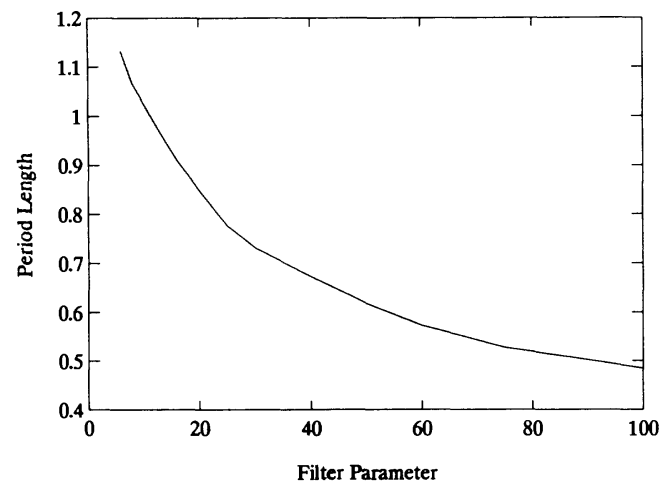

Figure 4a. Period Length at Optimum.

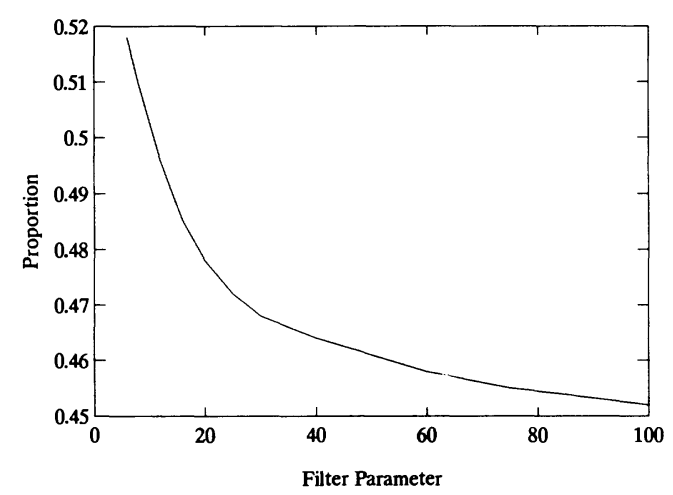

FIGURE 4c. Proportion of Up-Pulse at Optimum.

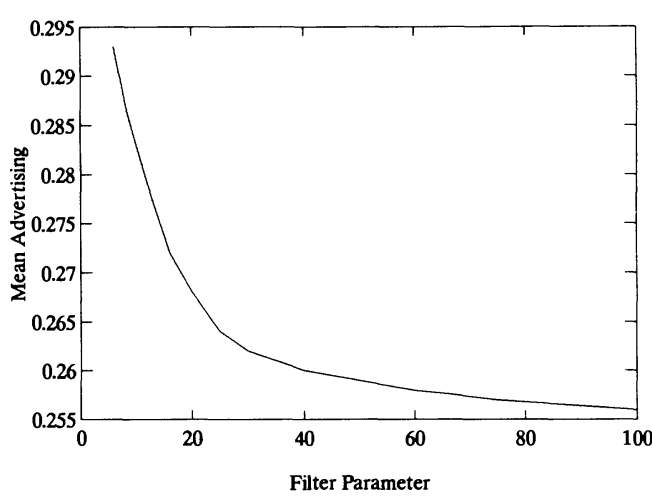

FIGURE 4b. Mean Advertising Level at Optimum.

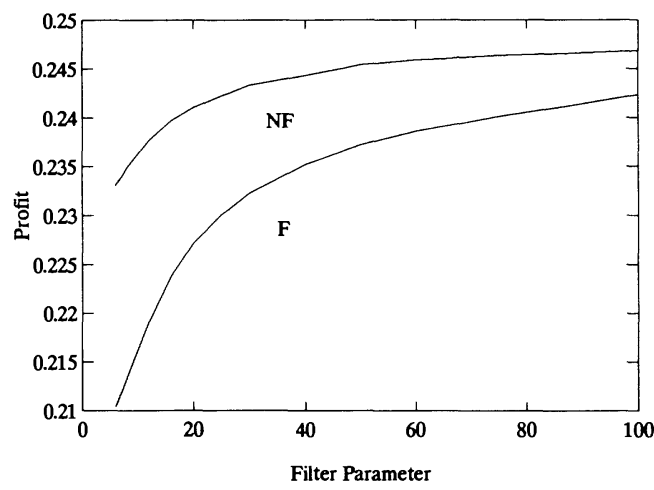

FIGURE 4d. Filtered and Nonfiltered Profit at Optimum.

pulse level $\bar{u}$ approaching the theoretical value 0.25 , the proportion $p$ spent on the upper pulse approaching the theoretical value $\frac{4}{9}$ and both nonfiltered and filtered profits approaching the theoretical maximum for the nonfiltered system, 0.25 . For smaller values of $\eta$, a pulsing input $u$ with small period length produces a nearly constant output $z$ of the same level, and fares worse than an appropriately chosen pulsing policy. Thus, $\tau, \bar{u}$ and $p$ all increase, while both nonfiltered and filtered profits decrease, the latter approaching the theoretical maximum for a constant input, 0.2098.

For values of $\eta$ less than those presented here, the filtered profit for the best pulsing policy can be so close to that for the best constant policy $(0.2098)$ that inaccuracies in simulating the differential system (27) make it difficult to determine if a pulsing policy can in fact be optimal. An additional grid search for $\eta=2$ gave strong evidence that, amongst rectangular policies, pulsing was not optimal, always inferior to the best constant spending policy. This offers evidence that the system (27) can admit pulsing optima only for sufficiently large values of the filter parameter $\eta$. It may be the case that, for values of $\eta$ below such a critical value, the optimal control properties of (27) are qualitatively similar to those of the same system with the filtering mechanism removed.

\section{Summary and Directions for Further Research}

An approach based on apparently logical assumptions about aggregate consumer response, that of diminishing marginal returns to advertising spending, tends to prescribe that the firm should spread its advertising funds as evenly as possible. If this strict concavity 
is relaxed, the optimal policy is to chatter. We have shown that, in the presence of an exponential filtering mechanism, such models' optimal policies may include pulsing.

Future research concerns the precise characterization of these optimal policies; what we've shown here is that they can be neither chattering nor constant spending. It is possible that optimal policies arising from filtered systems will not be of a rectangular (bang-bang) nature. If such policies are highly dependent on the specific functional form chosen for $g$, a unifying Sasieni-type analysis for filtered systems may not be possible.

The theoretical justification for the use of a filter, its appealing ability to make chattering and constant spending perform equally well, must be supplemented by empirical justification, perhaps experimentally. The partial adjustment mechanism suggested here, while plausible, is certainly not the only behavioral mechanism that could give rise to a filtered system; a variety of economic constructs yield more or less the same working equation as that used here to account for filtering. Further, even if a partial adjustment scheme were adopted, there is no reason to assume that it will operate in a manner that accords empirically with the filtering specification used in the class of models explored here.

To include filtering, models admired for their parsimony, like those of Vidale-Wolfe or Nerlove-Arrow, must allow for the estimation of a filter parameter as well, potentially complicating the estimation of other parameters and their interpretability. We have also made no attempt to identify appropriate econometric specifications, in terms of data and error structures, to be used in estimating second-order filtered models. Such complications, if they can be successfully resolved, must be weighed against the benefits of filtering in building aggregate continuous-time models of advertising response. ${ }^{7}$

Acknowledgements. The author would like to express his gratitude to John Little, for his help in every stage of this research, to John Hauser and the other members of this committee, Deborah Marlino and Don Kleinmuntz. Thanks also to Rick Staelin, Jim Bettman, Bill Boulding and two anonymous reviewers for their comments, and to the Business Associates Fund for its continuing support.

${ }^{7}$ This paper was received March 1990 and has been with the author 11 months for 2 revisions. Processed by Dominique M. Hanssens, former Area Editor.

\section{Appendix. Proof of Statements 1-3}

With $u$ and $z$ related by (15), the following holds at periodic equilibrium, proving statement 1 :

$$
0=z(\tau)-z(0)=\int_{0}^{\tau} \dot{z} d t=\int_{0}^{\tau} \eta(u-z) d t \Rightarrow \int_{0}^{\tau} z(t) d t=\int_{0}^{\tau} u(t) d t
$$

Solving ( 15 ) by an integrating factor yields the following:

$$
z(t)=z_{0} e^{-\eta t}+\eta e^{-\eta t} \int_{0}^{t} u(v) e^{\eta v} d v .
$$

As $t$ tends to infinity, the initial value $z_{0}$ is phased out. Using the fact that $z(t+\tau)=z(t)$ at periodic equilibrium, the long-run solution for $t \in[0, \tau]$ works out to be:

$$
z(t)=\frac{\eta e^{-\eta \tau}}{1-e^{-\eta \tau}} \int_{0}^{\tau} u(v+t) e^{\eta v} d v
$$

To prove statement 2, that the equilibrium path of $z$ approaches $u$ as $\eta$ increases, we rewrite (A-3) as:

$$
z(t)=\frac{1}{1-e^{-\eta \tau}} \int_{0}^{\tau} u(v+t) \eta e^{\eta(v-\tau)} d v .
$$

The external factor approaches 1 for large $\eta$. As for the integral, its value is computed as $u(t)$; this can be seen by considering $u$ constant (inside the integral) in a neighborhood of $\tau$, integrating conventionally, and taking the limit as $\eta$ approaches infinity. Thus, the equilibrium path of $z$ approaches $u$ on $[0, \tau]$. 
To prove statement 3 , that a chattering input $u$ to (15) causes a constant output $z$, we rewrite (A-3) as follows:

$$
z(t)=e^{-\eta \tau} \frac{\eta \tau}{1-e^{-\eta \tau}}\left[\frac{1}{\tau} \int_{0}^{\tau} u(v+t) e^{\eta v} d v\right] .
$$

Both the external factors above can be seen to approach 1 as $\tau$ approaches 0 , the second by applying l'Hôpital's rule. Considering the bracketed expression in the limit, the exponential factor approaches 1 uniformly on $[0, \tau]$, showing the integral to approach $\bar{u}$. Thus, as $u$ becomes a chattering policy, at periodic equilibrium $z$ will be constant.

\section{Bibliography}

Ackoff, R. L. and J. R. Emshoff (1975), “Advertising Research at Anheuser-Busch, Inc. (1963-1968),” Sloan Management Review, 16 (Winter), 1-16.

Bass, F. M. and D. G. Clarke (1972), "Testing Distributed Lag Models of Advertising Effect," Journal of Marketing Research, 9, 298-308.

Clarke, D. G. (1976), "Econometric Measurement of the Duration of Advertising Effect on Sales," Journal of Marketing Research, 13, 345-357.

Deal, K. R. (1979), “Optimizing Advertising Expenditures in a Dynamic Duopoly,” Operations Research, 27, 682-692.

Dhrymes, P. J. (1981), Distributed Lags, Amsterdam: North-Holland.

Eastlack, J. O. and A. Rao (1986), "Modeling Response to Advertising and Pricing Changes for V8 Cocktail Vegetable Juice," Marketing Science, 5, 3, 245-259.

Friedman, J. W. (1983), “Advertising and Oligopolistic Equilibrium,” The Bell Journal of Economics, 14, 464473.

Gould, J. P. (1970), “Diffusion Processes and Optimal Advertising Policy," in Microeconomic Foundations of Employment and Inflation Theory, E. S. Phelps et al. (Eds.), New York: W. W. Norton.

Hartl, R. F. (1987), “A Simple Proof of the Monotonicity of the State Trajectory in Autonomous Control Problems," Journal of Economic Theory, 41, 211-215.

Jones, P. C. (1983), “Analysis of a Dynamic Duopoly Model of Advertising," Mathematics of Operations Research, 8, 1, 122-134.

Koyck, L. M. (1954), Distributed Lags and Investment Analysis, Amsterdam: North-Holland.

Little, J. D. C. (1979), “Aggregate Advertising Models, The State of the Art,” Operations Research, 27, 629667.

Luhmer, A., A. Steindl, G. Feichtinger, R. Hartl and G. Sorger (1988), “ADPULS in Continuous Time," European Journal of Operational Research, 34, 171-177.

Mahajan, V. and E. Muller (1986), "Advertising Pulsing Policies for Generating Awareness for New Products," Marketing Science, 5, 2, 89-106.

Mesak, Hani I. (1985), “On Modeling Advertising Pulsing Decisions,” Decision Sciences, 16, $25-42$.

Monahan, G. E. (1983), “Optimal Advertising with Stochastic Demand,” Management Science, 29, 1, 106117.

Nerlove, M. (1958), Distributed and Demand Analysis, Agricultural Handbook No. 141, Washington, DC: USDA, June.

and K. J. Arrow (1962), “Optimal Advertising Policy Under Dynamic Conditions,” Economica, 29, $129-142$.

Nguyen, D. (1985), “An Analysis of Optimal Advertising Under Uncertainty,” Management Science, 31, 5, 622-633.

Ozga, S. (1960), “Imperfect Market through Lack of Knowledge,” Quarterly Journal of Economics, 74, 2952.

Rao, A. G. (1970), Quantitative Theories in Advertising, New York: John Wiley \& Sons, Inc. and P. B. Miller (1975), “Advertising/Sales Response Functions," Journal of Advertising Research, 15, 2, 1-15.

Rao, R. (1984), “Advertising Decisions in Oligopoly: An Industry Equilibrium Analysis," Optimal Control Applications and Methods, 5, 4, 331-344.

(1986), "Estimating Continuous Time Advertising-Sales Models," Marketing Science, 5, 2, 125-142.

Sasieni, M. W. (1971), "Optimal Advertising Expenditures," Management Science, 18, 64-72.

Sethi, S. P. (1973), "Optimal Control of the Vidale-Wolfe Advertising Model," Operations Research, 21, 9981013.

(1977), "Dynamic Optimal Control Models in Advertising," SIAM Review, 19, 4, 685-725.

(1979), "Optimal Advertising with the Contagion Model," Journal of Optimization Theory and Applications, 29, 4, 615-627. 
Simon, H. (1982), “ADPULS: An Advertising Model with Wearout and Pulsation,” Journal of Marketing Research, 19, 352-363.

Simon, J. L. and J. Arndt (1980), "The Shape of the Advertising Response Function," Journal of Advertising Research, 20, 11-28.

Steiner, R. L. (1987), "Point of View: The Paradox of Increasing Returns to Advertising," Journal of Advertising Research, 27, 45-53.

Tapiero, C. S. (1978), "Optimal Advertising and Goodwill under Uncertainty," Operations Research, 26, 450463.

(1979), "A Generalization of the Nerlove-Arrow Model to Multi-Firm Advertising under Uncertainty," Management Science, 25, 9, 907-915.

(1982), “A Stochastic Model of Consumer Behavior and Optimal Advertising," Management Science, $28,9,1054-1064$.

Thompson, G. L. (1981), “An Optimal Control Model of Advertising Pulsation and Wearout," Marketing: Measurement and Analysis, Proceedings of the Third ORSA / TIMS Special Conference on Market Measurement and Analysis, J. W. McKeon (Ed.), 34-43.

Vanhonacker, W. R. (1983), "Carryover Effects and Temporal Aggregation in a Partial Adjustment Model Framework," Marketing Science, 2, 3, 297-317.

Vidale, M. L. and H. B. Wolfe (1957), "An Operations Research Study of Sales Response to Advertising," Operations Research, 5, 370-381.

Zielske, H. A. (1959), "The Remembering and Forgetting of Advertising," Journal of Marketing, 23 (January), 239-243. 